\title{
TYPE III RADIO BURST PRODUCTIVITY OF SOLAR FLARES
}

\author{
M. Poquerusse ${ }^{1,2 *}$, P.S. McIntosh \\ 1 NOAH/ERL/SEL, 325 Broadway, Boulder CO 80302, USA \\ 2 Observatoire de Mieudon, 92105 MIEUDON PRINCIPAL CEDEX, FRANCE \\ * Natioual Research Council - NOAA research associate
}

We study the statistical relationship between optical flares and type III radio bursts, using modern and extensive computer files. Results emerge along two main lines, concerning the physical mechanism of ejection of energetic particles, and the magnetic field geometry respectively.

First, we find that type III probability of occurrence increases strongly with the brightness of a flare and its proximity to a sunspot, and with accompanying prominence activity. This suggests that Bornmann's class I and III events correspond to distinct physical phenomena, particle acceleration and magnetic expansion respectively, both working simultaneously in class II events, which are the most favorable to the ejection of energetic particles out of flaring sites.

Second, we find that type III probability is maximum for flares at about 30 degrees east of the central meridian and one degree west of the center of the main group of flares in a given active region. We conclude that field lines on the west side of an active region are the most likely to be open onto the interplanetary medium and that they are on the average tilted 30 degrees westward.

\section{OBSERVATIONS AND FLARE-TYPE III ASSOCLATION CRITERION}

Optical flares : NOAA/NESDIS/NGDC comprehensive computer files generated by Helen Coffey and John McKinnon in Boulder, Colorado, from world-wide reports.

Type III bursts: ARTEMIS digital multichannel radiospectrograph in Nancay, France (500-100 MIIz).

From the crosscorrelation function of flare and type III burst occurrence times, we find, in basic agreement with Hughes and Harkness (1963, Astrophys. J.138,239), that type III bursts correhate best with the time of the maximum of the flare as observed in Ha. We make use of the position and width of the peak in the crosscorrelation function to select the time difference between flare maximum and type III burst that is typical of an association, and then select a set of flares during the period 1980-1987 for further study, with the following characteristics:

occur in interval 6-18h UT, when Nancay can observe

no other flare within $10 \mathrm{~min}$ of maximum

dispersion of reported maximum times $\leq 3 \mathrm{~min}$

available reports of $\mathrm{Ha}$ brightness (faint $\mathrm{F}$, normal $\mathrm{N}$, bright $\mathrm{B}$ )

We then distinguish type III associated (T-III) from non-associated (NT-III) flares by the time interval from flare maximum to burst:

\begin{tabular}{c|ccccc} 
flare class & type IIIs & time interval & $\rightarrow$ & number of cases & code \\
\hline \multirow{2}{*}{ NT-III } & none & $-10+10 \mathrm{~min}$ & 7906 & - \\
T-III & 1 or more & $-2+1 \mathrm{~min}$ & 190 & $\ldots .$.
\end{tabular}

The rate of error in class identification (NT-III instead of T-III and vice versa) is about $15 \%$.

On the figures presented, we will give error bars for the T-III flares (square root of the number in bin); error bars for the (much more numerous) NT-IIl flares have much smaller relative values. 

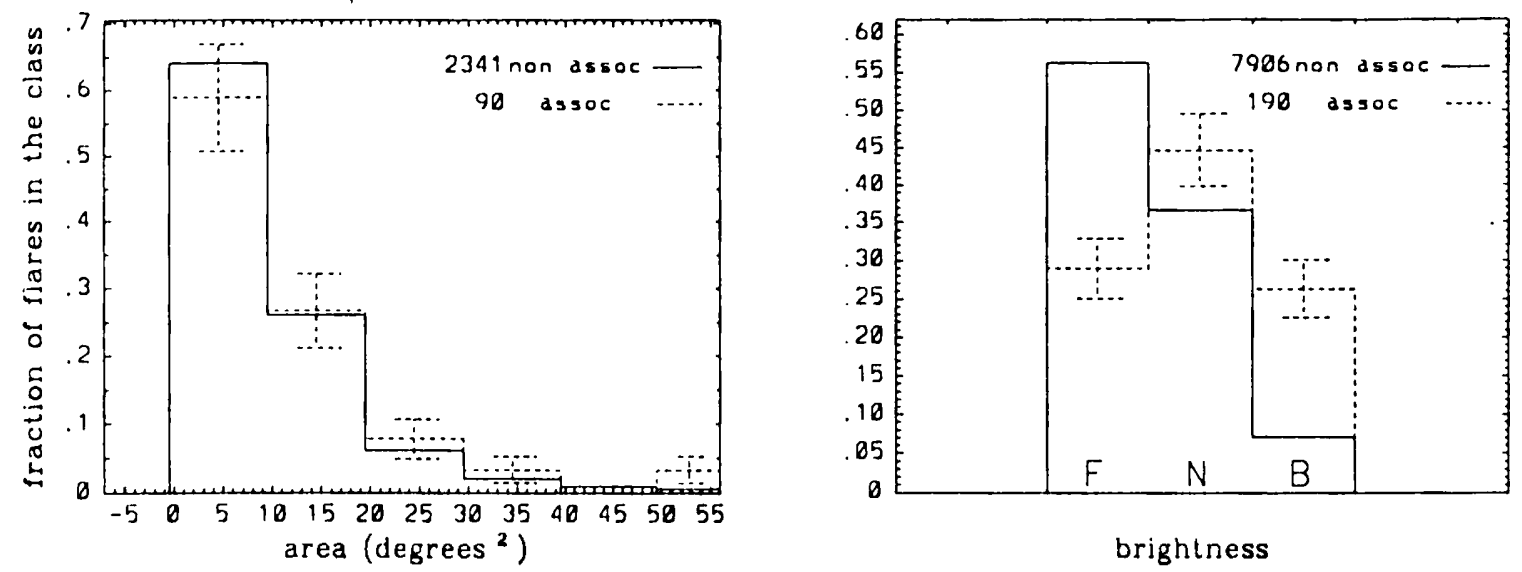

At variance with previous results (Kundu: 1965, Solar Radioastronomy), we find a weak dependence of type III association rate on flare area and a strong dependence on flare brightness. It is likely that the Ha intensity is correlated to the energy of the particles trapped in the core magnetic loops, which thus appears to be a dominant factor in the release of energetic electrons on open magnetic field lines.

\section{DEPENDENCE ON PROMINENCE ACTIVITY AND PROXIMITY TO SUNSPOTS}

Observers sometimes inclucle remarks in their reports of optical flares (LAU standard list), coded A through $\mathrm{Z}$ in the NGDQ flare lists. Four remarks concerning associated phenomena occur frequently:

$\mathrm{G}=$ no visible spots nearby $\mathrm{H}=$ flare accompanied by high-speed dark filament

$\mathrm{L}=$ existing filaments suddenly active $\mathrm{Z}=$ major sunspot umbra covered by flare These remarks allowed us to divide the flares according to the degree of prominence/filament activity and of spot proximity, albeit in a crude sense (these remarks are subjective and are not included by all observers). The following tables give the numbers of flares in each category, and reveal trends in the association with type III bursts as a function of both associated phenomena. We have fitted a "log-linear model" to these contingency tables (Bishop et al:: 1975, Discrete Multivariate Analysis) and we give the resulting cross product ratios at fixed flare brightness.

\begin{tabular}{c|crrr} 
PROMINENCE ACTIVITY & class & F & N & B \\
\hline low & NT-III & 4315 & 27.45 & 526 \\
(neither remark H nor L) & T-III & 50 & 78 & 43 \\
\hline ligh & NT-III & 138 & 150 & 32 \\
(remarks II or L or both) & T-III & 5 & 7 & 7
\end{tabular}

(T-III/NT-III at high prom. activ.) / (T-III/NT-III at low prom. activ.) $=2.2$

\begin{tabular}{c|crrr} 
PROXIMIITY TO SPOTS & class & F & N & B \\
\hline low & NT-III & 63 & 61 & 7 \\
(remark G) & T-III & 0 & 1 & 0 \\
\hline medium & NT-III & 4388 & 2821 & 542 \\
(neither G nor Z) & T-III & 55 & 83 & 48 \\
\hline high & NT-III & 2 & 13 & 9 \\
(remark Z) & T-III & 0 & 1 & 2
\end{tabular}

(T-III/NT-III close to spot) / (T-III/NT-III far from spot) $=7.9$ 
These results confirm the dependence of type III occurence on prominence activity and surges (Svestka: 1976, Solar Flares), and on proximity to sunspot (Zanelli et al.: 1980, Solar Phys. 65,387). In addition, we find that this is not a mere consequence of common dependences on flare brightness: flare - type III association rate increases with proximity to sunspot and with prominence activity at fixed flare brightness. These two criteria correspond to opposite ends in Bornmann's event classification (1987, BAAS 18,898), class I (compact flares) and class III (mass cjections), suggesting that both low-altitude electron acceleration in strong fields and breaking of magnetic field lines are favorable factors to the release of energetic electrons into the corona and the solar wind. This leads to the following picture. Class I events accelerate particles in abundance, confined at low altitude by the closed and stable field lines, while class III events consist in magnetic expansion into the interplanetary medium without necessarily much particle acceleration. Only when both circumstances occur together do we have ejection of energetic electrons into the interplanetary medium. This happens only in composite events (class II ?).

\section{DEPENDENCE ON THE DISTANCE TO THE CENTRAL MERIDIAN AND ON THE POSITION IN THE ACTIVE REGION}

DISTRIBUTION OF FLARES ACROSS THE DISC

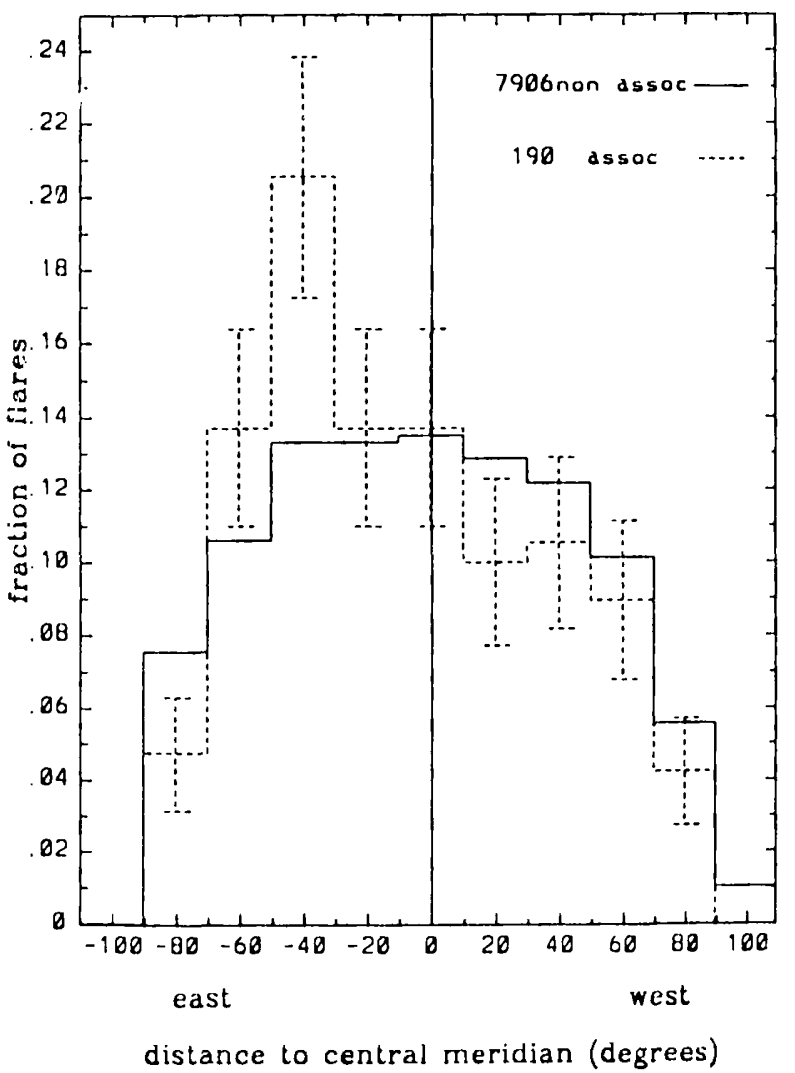

AUTOCORRELATION OF THE NUMBER OF FLARES AS A. FUNCTION OF CARRINGTON LONGITUDE AND CROSSCORRELATION OF THE 2 CIASSES

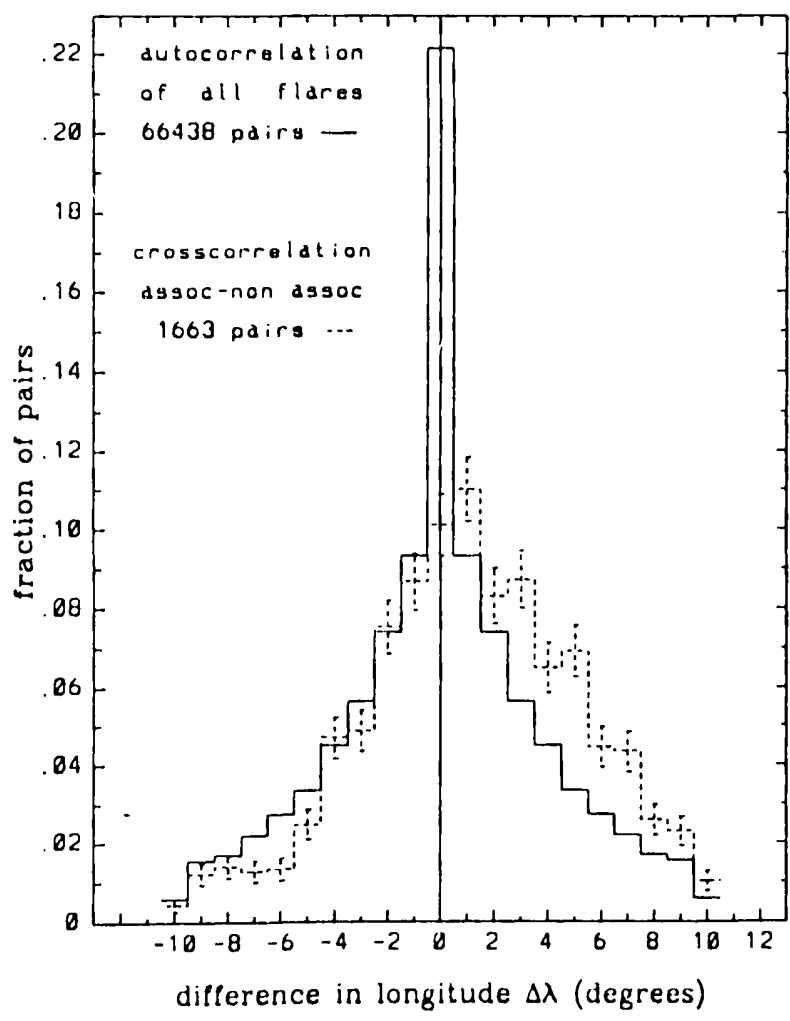

for crosscorrelation, $\Delta \lambda=\lambda_{\text {a..oc }}-\lambda_{\text {non aud }}$

The figure on the left shows that the E-W distribution of T-III flares presents a broad maximum around 30 degrees east of the central meridian. Malville (1962, Astrophys. J. 135,83-1) reported an eastern excess of type III associated flares, but it was at the limit of statistical significance and had never been confirmed since. We conclude that type III radiation patterns are on the average tilted by about 30 degrees west of the radial, which we interpret by a corresponding tilt of open magnetic field lines at the base of the corona. These open lines will be pulled back to the radial by the solar wind, at about 1 solar radius above the photosphere and 15 degrees west of the liare site. 
The crosscorrclation function in Carrington longitude $\lambda$, shown in dashed line on the bottom right figure of the previous page, includes all T-III - NT-III pairs of flares with differences in time $\leq 3$ days, and differences in latitude $\leq 5$ degrees. It is assymmetrical compared to the origin $\Delta \lambda=0$, showing that in active regions, the class of T-III flares is on the average displaced westward by about 1 degree relatively to the class of NT-III flares. This is consistent with the fact that type III polarization is generally determined by the magnetic polarity of the leading sunspot (Suzuki, Dulk: 1985, in Solar Radiophysics, p 318), and with Jackson's observation (1986, Solar Phys. 105, 123) that in simple bipolar regions type III positions are shifted westward of the center of the optical active region. This is also reminiscent of the fact that surges occur more of ten on the west side of active regions. We conclude that magnetic field lines are more likely to be open on the west side than on the east side of an active region.

It thus appears that both the orientation and the position of open field lines in active regions present an average east-west asymmetry, which is schematically represented below.

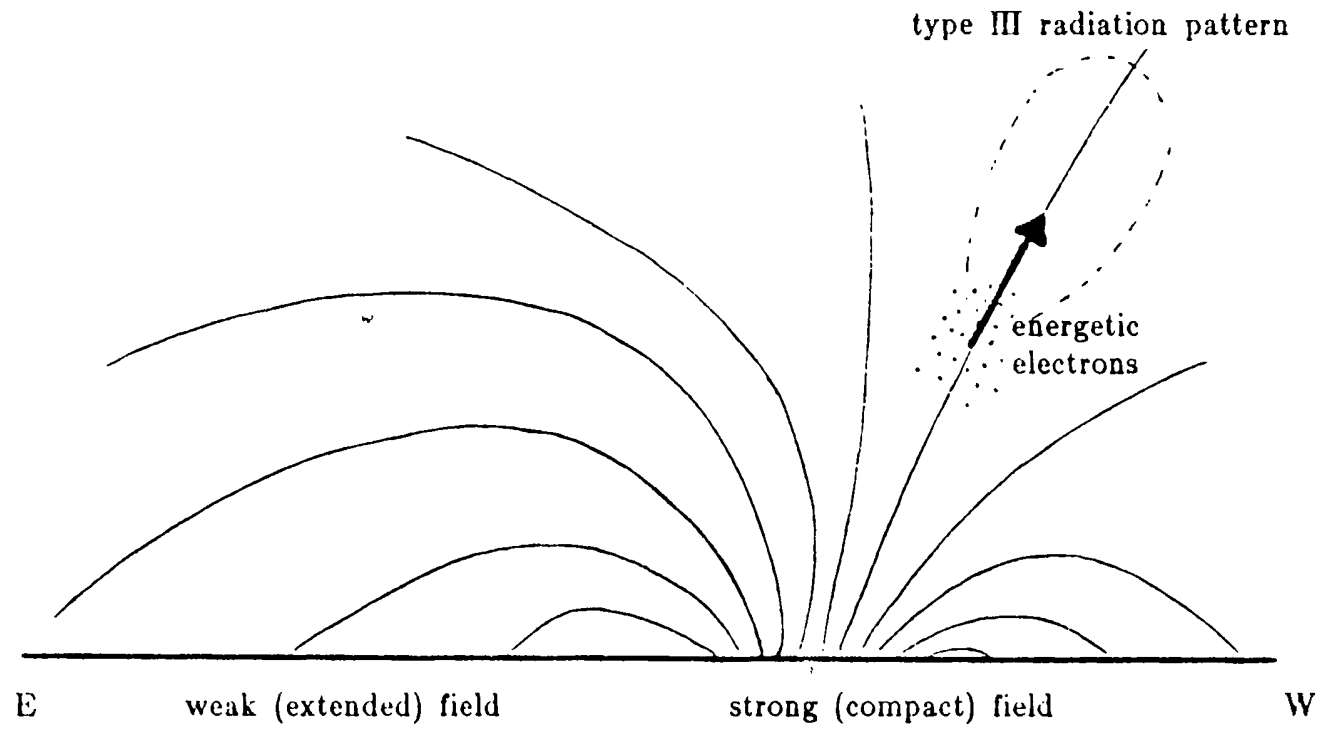

It seems that the highest coronal loops emerging from an active region, which are the most likely to give rise to open field lines, connect the leading polarity inside the active region to the opposite polarity on the photosphere outside and east of the region. This is probably a consequence of the fact that the leading (polarity) part of an active region covers a smaller area on the photosphere, with bigger spots, than the trailing part.

\section{CONCLUSION}

First, the behavior of type III occurrence probability during a flare, as a function of associated phenomena, suggests that Bornmann's event classification corresponds to varying amplitudes of 2 basic mechanisms, particle acceleration and magnetic expansion.

Second, the distribution of type III producing flares, across the disc and in Carrington longitude, points to a general east-west assymmetry of the magnetic field in the corona above active regions, which reflects the well known assymmetry between leading and trailing sunspots. An interesting consequence is that interplanetary streams of energetic particles coming Irom flares may be centered about 15 degrees west of the flare longitude. 